\title{
Une cité carnavalesque?
}

Le carnaval de Notting Hill à l'épreuve des politiques de développement urbain

A carnivalesque city? Notting Hill Carnival put to the test by politics of urban development

\section{Lionel Arnaud}

\section{(2) OpenEdition}

\section{Journals}

Édition électronique

URL : http://journals.openedition.org/plc/830

DOI : $10.4000 /$ plc. 830

ISSN : 2117-5209

Éditeur

L'Harmattan

Édition imprimée

Date de publication : 1 janvier 2010

Pagination : 163-187

ISBN : 978-2-296-1141-3

ISSN : $1279-8657$

\section{Référence électronique}

Lionel Arnaud, "Une cité carnavalesque ? », Pouvoirs dans la Caraïbe [En ligne], 16 | 2010, mis en ligne le 06 mai 2011, consulté le 14 novembre 2019. URL : http://journals.openedition.org/plc/830 
UNE CITE CARNAVALESQUE ?

LE CARNAVAL DE NOTTING HILL A L'EPREUVE DES POLITIQUES DE DEVELOPPEMENT URBAIN

\author{
Lionel ARNAUD \\ Centre de recherches sur l'action politique \\ en Europe (CRAPE) \\ Université Rennes 1
}

Depuis la fin des années 1970, le carnaval de Notting Hill a souvent été présenté comme «un baromètre des tensions urbaines et des relations raciales» (The Independant, $1^{\mathrm{er}}$ août 1988) et «le terrain sur lequel des attitudes rivales à l'égard de la race en Grande-Bretagne, des conceptions rivales de la «culture nationale» se combattent» (New Statesman and Society, 26 août 1988). Dans le même temps, la métaphore du carnaval était mobilisée par les théoriciens de la «nouvelle gauche» pour tenter de comprendre les transformations sociale et culturelle de la société britannique. A la place de l'opposition binaire de la «lutte des classes » où les transformations sociales, symboliques et culturelles étaient conçues ou imaginées de façon globale, les chercheurs du Centre for Contemporary Cultural Studies (CCCS) de Birmingham, dont l'influence intellectuelle a été déterminante sur la gauche radicale (Kenny 1995), ont présenté le carnaval comme un espace de négociation où les positions de résistance deviennent stratégiques et sont elles-mêmes produites par les structures du pouvoir ${ }^{1}$. Parvenues au pouvoir de 1980 à 1985 avec l'élection de Ken Livingston à la tête du Great London Council (GLC) et de la Great London Authority (GLA) à partir de 2000, les idées de la New Left ont ainsi contribué à réinventer le carnaval de Notting Hill pour tenter de gérer la complexité du multiculturalisme et de la globalisation au niveau local (Bianchini 1989).

Mais quelles sont les conséquences de cette transformation du rôle et de la place du carnaval sur les politiques publiques? Les enjeux du carnaval de Notting Hill demeurent plus particulièrement liés aux luttes et aux revendications des minorités ethniques afro-antillaises. Après deux décennies de répression, la progressive reconfiguration des enjeux urbains accompagne-t-elle et favorise-t-elle une nouvelle justification du carnaval, et

\footnotetext{
${ }^{1}$ Souvent cité par les cultural studies britanniques, le travail de Mickael Bakhtin sur la suspension temporaire de l'ordre moral et symbolique accepté dans les foires, les festivals, les mardi gras et les autres formes de festivités populaires du Moyen Age suggère que les signes n'ont pas de signification fixe; le sens est généré au sein d'une relation double, entre le locuteur et l'auditeur, l'acteur et le spectateur. Cf. Bakhtin 1970.
} 
finalement des minorités ethniques, dans la cité dite «globale» (Sassen 1991) ? Assiste-t-on à une nouvelle mise en forme des dispositifs de mobilisation dans le sens d'une "cité carnavalesque », un univers de justification censé adoucir les critiques culturelles formulées par les minorités ethniques contre le système politique, les forces politiques valorisant aujourd'hui le carnaval pour mieux promouvoir une société multiculturelle, les entreprises pour exploiter sa dimension créative et les mouvements identitaires pour démontrer le caractère hybride de la société britannique?

En nous fondant principalement sur les discours et les débats qui ont accompagné le développement du carnaval depuis la fin des années $1970^{2}$, l'enjeu de cette contribution est de procéder à un examen des processus sociaux qui président aujourd'hui à la promotion sociale et culturelle du carnaval de Notting Hill par le gouvernement local, en présentant d'une part, les affrontements d'acteurs auxquels le carnaval a donné lieu et en examinant, d'autre part, les médiations par lesquelles les références à la « cité globale » (qui désigne ici non seulement l'entité urbaine formée par la ville de Londres, mais plus largement l'univers qui lui sert aujourd'hui de justification) permettent le passage du minoritaire au bien commun, du particulier au général et finalement la légitimation du carnaval comme outil au service du développement social de la ville de Londres (Botanski et Thévenot 1991, Lafaye et Thévenot 1993).

\footnotetext{
${ }^{2}$ Cette contribution s'inscrit dans un travail plus vaste, débuté en 2000 et visant à comparer les dynamiques idéologiques et sociologiques qui ont contribué à intégrer, en Angleterre et en France, certaines pratiques culturelles comme le carnaval et le hip hop dans les stratégies de développement et de communication des villes européennes. Nous utilisons ici principalement des données tirées de l'analyse du contenu des journaux quotidiens britanniques parus depuis 1975, notamment à partir des archives du Centre for Research in Ethnic Relations (CRER) de l'université de Warwick. Contrairement à de nombreuses analyses des informations traitées dans les médias, nous ne nous intéressons pas principalement à la manière dont les médias cadrent les événements, mais à la manière dont sont relatées dans les médias la mobilisation, les déclarations publiques et autres formes de revendications des acteurs non médiatiques. L'ensemble des données exclut les revendications faites hors de la sphère publique (par exemple les pressions exercées de manière interne), de même que les revendications dans des sphères semi-publiques (par exemple les demandes faites au sein des espaces de consultation, ou les questions purement locales). Néanmoins, il faut préciser que, une fois que ces revendications deviennent controversées, on a tendance à en parler dans la presse nationale et donc nos sources les mentionneraient.
} 


\section{Le carnaval comme enjeu de politique publique}

Examinons tout d'abord la confrontation des différents acteurs autour et dans le carnaval. Si l'angle de l'action stratégique est pertinent pour comprendre les jeux de pouvoir entre les différents acteurs (Gutzmore 1993 ; Pryce 1995), il ne permet pas de saisir ce que le carnaval autorise en passant du particulier au général. De son côté, la sociologie des mouvements sociaux a perçu la capacité du carnaval de Notting Hill à construire un mouvement collectif sur des positions critiques qui se réfèrent à l'identité (Cohen 1991 ; 1993). Mais quelles sont exactement les formes du collectif qui sont constituées de cette façon ? Sont-elles identiques à celles créées par une solidarité autour des mêmes droits pour tous ? Avant d'analyser ces questions, il est important de situer correctement les arguments des différents acteurs pour mieux mesurer la portée de la question tant au niveau local que national.

\subsection{Les différentes orientations des associations de défense du} carnaval

A l'image d'une «communauté afro-antillaise » regroupant des populations très diverses, ne serait-ce qu'en raison de leurs origines nationales (Trinité-et-Tobago, Jamaïque, Barbade, Sainte-Lucie...), les associations qui ont présidé à l'organisation et à la défense du carnaval de Notting Hill depuis la fin des années 1960 expriment des positions très différentes. Leurs points de vue peuvent toutefois être regroupés autour de trois orientations-types : expressive, économique et communautaire, qui se manifestent non seulement via les raisons évoquées et les arguments développés mais aussi par le type de ressources disponibles.

A travers les cinq disciplines représentées dans le carnaval de Notting Hill : les mas (costumes, masques), les steel bands, le calypso (la musique traditionnelle du carnaval de Trinité-et-Tobago), la soca (une fusion de soul et de calypso) et les sound systems (qui jouent différents styles de musique contemporaine comme le reggae, le dub, le hip-hop, la soul et le r'n'b, le garage et le 2-steps, la jungle et le drum'n'bass...), le potentiel artistique du carnaval est un enjeu fondamental pour les organisateurs qui y ont très tôt perçu un élément de légitimation culturelle. Pendant longtemps, ce fut d'ailleurs l'un des principaux arguments du Carnival and Arts 
Committee (CAC) qui a présidé aux destinées du carnaval de 1977 à 1988. Sous l'autorité de Victor Crichlow et d'Alex Pascall, le carnaval a ainsi été développé comme un événement susceptible de contribuer à l'expression artistique des afro-antillais de Londres. Au fil des années, plusieurs organisations se sont en outre formées pour améliorer les rapports entre les organisateurs et promouvoir leur discipline d'un point de vue artistique : l'Association of British Calypsonians, la Caribbean Music Association, la British Association of Steel Bands, la British Association of Sound Systems... Ces associations faisaient partie intégrante du conseil d'administration du Notting Hill Carnival Trust (NHCT), la nouvelle structure juridique chargée de gérer le carnaval depuis 1988.

Car si le carnaval demeure d'abord pensé comme un événement culturel, il n'échappe pas aux pressions de la rentabilité. En mars 1989, face à plus de 200000 livres de dette, le CAC qui avait piloté le carnaval pendant 12 ans, annonce sa liquidation sur les recommandations d'un audit ordonné par son conseil d'administration. Réalisé par les consultants Cooper \& Lybrand, cet audit soulignait que les organisateurs avaient constamment ignoré les attentes de la «communauté noire », négligeant les opportunités susceptibles de rendre l'événement profitable à tous. Des changements radicaux sont alors demandés, en premier lieu le remplacement de l'ancienne équipe et l'introduction d'une culture d'entreprise d'inspiration thatcherienne. Une nouvelle structure est créée, la Notting Hill Carnival Entreprise (NHCE) où la représentation de la communauté locale au sein du Conseil d'administration est statutairement garantie à $60 \%$, et à la tête de laquelle est élue une jeune avocate britannique d'origine trinidadienne, Claire Holder. Cette dernière engage immédiatement un certain nombre de réformes visant à rendre l'événement non seulement rentable, mais également profitable. En avril 1997, l'organisation met clairement en avant ses orientations managériales en se transformant en Notting Hill Carnival Trust (NHCT). Proclamant de son côté la dimension «purement» artistique du carnaval, le CAC tente un temps de poursuivre ses activités tandis que deux associations sécessionnistes débutent une campagne virulente contre le NHCT : le Notting Hill International Carnival Committee et l'Association for a People's Carnival.

A ce niveau, il est important de rappeler que le carnaval fut créé au lendemain des émeutes anti-noirs qui avaient violemment agité le quartier de 
Notting Hill pendant l'été de 1958 (Pilkington 1988). Convaincue de la nécessité de rassembler la communauté noire et d'encourager une meilleure entente avec les autres habitants, la directrice de la West Indian Gazette, Claudia Jones, prend l'initiative, le 30 janvier 1959, d'organiser le premier carnaval de Londres dans la salle des fêtes de St Pancras. Mais c'est surtout dans les années 1970 que le carnaval prend véritablement de l'ampleur avec le succès du reggae et l'introduction concomitante des sound systems qui contribuent à attirer la jeunesse tout en soulignant la dimension politique de l'événement. De fait, des affrontements très violents avec la police ne tardent pas à se produire, d'abord en 1976, puis tout au long des années 1980. Ajoutées aux émeutes dites «raciales » de Toxteth et Brixton de 1981, ces violences endommagent durablement l'image de la communauté afroantillaise et sa capacité à gérer un événement de cette ampleur. Après une décennie de division, les leaders afro-antillais tentent alors de s'entendre pour améliorer l'organisation d'un événement devenu gigantesque : «Il y a eu un changement d'attitude, expliquait Alex Pascall. Nous avons réalisé que nous ne connaissions pas le système. Ce n'était pas bon de rester sur la touche. Nous devions rentrer dans le jeu. Toxteth et Brixton nous ont fait comprendre que quelque chose devait être fait» (cité par The Observer, 29 août 1982). La nécessité de s'auto-organiser pour défendre les droits des Afro-Antillais et combattre le racisme est d'ailleurs restée au centre de l'argumentaire de Claire Holder lorsqu'elle prend en main l'organisation du carnaval en 1989 : «La perpétuation du mythe d'une race de personnes inférieures forcément engagées dans des activités criminelles ou avec des standards très faibles d'éducation et des modes de vie indissociables de leur culture est, pour le dire une bonne fois, insultante. Et lorsque nous, les noirs, acceptons ce type de non sens et que nous ne cherchons pas, ne créons pas et ne saisissons pas les opportunités de notre propre développement, c'est encore plus insultant » (cité par The Caribbean Times, 25-31 août 1989).

\subsection{Les différents langages des autorités de tutelle du carnaval}

Le caractère fragmenté des administrations politiques est un phénomène bien reconnu aujourd'hui, et le carnaval est un parfait exemple de la variété des porte-paroles publics qui, de l'Arts Council à la Metropolitan Police, de la Commission for Racial Equality (CRE) au Royal Borough of Kensington \& Chelsea, interviennent dans son organisation. 
L'analyse de l'action publique a mis en lumière la capacité d'adaptation et d'arrangement des fonctionnaires vis-à-vis de leurs interlocuteurs extérieurs (Hassenteufel 1995), ou encore l'existence de sous-systèmes politiques au sein de l'administration (McCool 1989). Nous nous contenterons pour notre part d'étudier les systèmes de pensée et d'action, l'analyse des langages principaux, des référentiels et des paradigmes utilisés par les autorités (Howlett 1994 ; Jobert et Muller 1987). Concernant le carnaval de Notting Hill, nous pouvons ainsi identifier trois « langages » : sécuritaire, managérial et multiculturaliste, tour à tour utilisés par les élus et les professionnels.

Depuis 1976 et les dizaines de blessés et de dégâts matériels occasionnées par les confrontations entre les jeunes Antillais et la police, les autorités publiques locales et nationales n'ont quasiment jamais cessé de percevoir avec suspicion le carnaval de Notting Hill, au point de mettre en place un dispositif quasi militaire de surveillance et de contrôle. La zone est quadrillée aujourd'hui en six sections où la circulation automobile est interdite et les résidents soumis à un contrôle d'identité. Certaines rues sont fermées et les places de parking extrêmement réglementées. Depuis le milieu des années 1980, des caméras vidéo ont été installées tandis que des hélicoptères surveillent la zone et que des policiers spécialisés dans l'arrestation des vendeurs de drogues et de fauteurs de trouble sont déployés parmi les festivaliers. Depuis 1987, un couvre feu a été instauré après vingt heures trente étant entendu que, selon la police, «beaucoup de crimes et de désordres qui entachent la réputation du carnaval surviennent après la tombée de la nuit » (cité par The Times, 17 août 1988). Un des premiers rôles du Royal Borough of Kensington and Chelsea et du Westminster City Council est en outre «de s'assurer que l'événement est organisé en accord avec les règles d'hygiène et de sécurité en vigueur au regard du commerce de rue (licence et hygiène alimentaire) et des «sound systems » (niveau de bruit et nuisance sonore) » (The Royal Borough of Kensington and Chelsea, 2000, p. 1).

Parallèlement à cette politique fondée sur «la loi et l'ordre» caractéristique de l'attitude de Margaret Thatcher à l'égard des minorités ethniques, les autorités se sont efforcées de rentabiliser le carnaval en imposant plus ou moins directement un processus de managerialisation de son organisation. Au lendemain de la publication du rapport de Cooper \& Lybrand en 1988, un certain nombre de bailleurs de fonds se sont d'abord 
détournés du CAC en raison de sa «mauvaise gestion» du carnaval. L'année suivante, le carnaval ne perçu qu'un tiers des $£ 300000$ obtenues en 1988 : le Kensington and Chelsea Council fit ainsi passer sa contribution de $£ 55510$ à $£ 17730$, tandis que le CRE n'accordait plus qu'un quart de sa subvention de 1988 (soit environ $£ 5$ 000) et que l'Arts Council divisait pour sa part sa contribution de moitié pour la porter à $£ 50$ 000. La même année, l'anxiété des sponsors conduisit à annuler $90 \%$ des festivités précédant le carnaval. L'objectif manifeste de toutes ces pressions peut être clairement relié aux pressions du gouvernement visant à limiter au maximum les dépenses publiques, en particulier dans des secteurs jugés «improductifs». Le leader du groupe conservateur au Westminster City Council, Lady Porter, ne tarda pas ainsi à demander au CAC le remboursement de la somme de $£ 20000$ pour couvrir les dépenses publiques engagées dans l'organisation logistique de la manifestation (mise en place de toilettes mobiles et de nouveaux éclairages, fermeture des routes, protection des jardins, nettoyage des rues et ramassage des ordures). Progressivement, ces pressions favorisèrent l'ingérence des pouvoirs publics dans l'organisation et la gestion du carnaval, la mise en place du NHCT ayant été quasiment imposée par le gouvernement local.

Mais tandis que le carnaval devenait, au début des années 1990, un événement suffisamment rentable et sécurisé, les principales personnalités politiques se tournaient désormais vers Notting Hill pour vanter les mérites d'une société multiculturelle. A commencer par la Reine elle-même qui, dès 1988 et à l'occasion d'une visite à Trinité-et-Tobago, n'hésite pas à présenter le carnaval de Notting Hill comme «our carnaval» (cité par The Voice, 18 août 1990). Pour le Premier ministre John Major, le carnaval « nous offre l'opportunité d'être fier à juste titre de notre diversité culturelle qui enrichit notre patrimoine national » (cité par The Daily telegraph, 31 août 1992), tandis que dans une lettre au chef de Scotland Yard, le ministre de l'Intérieur Douglas Hurd se réjouissait du « retour du véritable esprit du Carnaval - une célébration pacifique et joyeuse de personnes de toutes les races et de tous les milieux » (cité par The Daily Telegraph, 31 août 1988). Mais l'instrumentalisation la plus provocante du carnaval fut sans aucun doute la visite très médiatisée du leader conservateur William Hague et de sa fiancée le 26 août 1997, qui n'ont pas hésité à venir «visiter» le carnaval en tenue de safari... De fait, cette approche apparemment plus conciliante des Conservateurs à l'égard d'un carnaval qu'ils n'ont jamais cessé de dénigrer 
et de combattre, cache finalement mal un désir de se réapproprier un événement désormais incontournable et internationalement célèbre.

\subsection{Les contraintes des groupes de pression}

La politique du carnaval ne se résume pas à un face à face entre des organisateurs et des autorités publiques. D'autres acteurs influencent les termes du débat en tentant de rattacher leurs actions et les intérêts qu'ils défendent à une forme de bien commun (Offerlé 1998). Directement ou par la médiation de «représentants» (le plus souvent autoproclamés), ces groupes font pression sur les organisateurs et les autorités pour faire entendre leur voix singulière tout en proclamant une forme de propriété collective.

Depuis 1976, le lien entre les jeunes noirs et les violences urbaines est en particulier une équation quasi automatique des commentaires publiés par la presse populaire et une des principales préoccupations des autorités et des organisateurs du carnaval. La volonté des autorités locales de les éloigner du carnaval a cependant toujours été sévèrement critiquée par la plupart des militants «communautaires », qui se sont souvent servis de ces violences pour légitimer une approche politique de l'événement (Arnaud 2005). Le CAC a ainsi toujours refusé de condamner les gangs noirs lorsqu'ils étaient accusés d'avoir déclenché les batailles de rue de 1976, 1987 et 1988. Pour Louis Chase, qui présidait aux destinées du CAC au lendemain des émeutes de 1976, la conscience politique que les jeunes afroantillais ont suscité dans le carnaval, en tant qu'événement culturel noir de masse, réside dans la défense d'une certaine sous-culture de rue; cette dernière étant entrée en résistance contre la domination culturelle de la société blanche (Chase 1978). En ce sens, et comme le réaffirmait plus récemment le militant afro-antillais Cecil Gutzmore à propos de la violence au sein du carnaval, «le vol par les jeunes noirs dans la société ne peut être apolitique, parce qu'il est le produit du fonctionnement de la société capitaliste (de laquelle ils sont massivement exclus) et de sa culture individualiste » (Gutzmore 1993, p. 229). De ce point de vue, le carnaval est perçu par certains militants communautaires comme un moyen de réaliser le pouvoir des masses noires en tant que minorité subordonnée luttant pour son émancipation des structures profondément racistes de la société britannique. 
L'interdiction du carnaval de façon à mettre un terme définitif à ses débordements fut en outre un sujet particulièrement discuté lors de la campagne municipale de 1988. Les élus locaux conservateurs (majoritaires au conseil municipal de Kensington \& Chelsea) ne manquèrent pas de relayer le ressentiment de certains de leurs administrés à l'égard des débordements occasionnés par cet événement. Avec la gentrification progressive du quartier (i.e. le remplacement progressif des classes populaires par des classes moyennes ou supérieures), les résidents sont de fait apparus de moins en moins tolérants à l'égard du carnaval, lassés d'abandonner leur maison pendant trois jours, dégoûtés de voir leurs entrées et leurs jardins transformés en urinoirs, exaspérés par la suppression des bus, des taxis, alors même que les rues étaient envahies par une foule « incontrôlable et dangereuse »... De la dénonciation des désordres au racisme, il n'y a qu'un pas que certains résidents (souvent relayés par le parti conservateur) n'ont parfois pas hésité à franchir, à l'image de cet écrivain d'une quarantaine d'année interviewé par The Daily Telegraph (30 août 1998): "Ils nous demandent de les accepter eux et leur mode de vie. J'aimerais que le même principe soit respecté en retour. Je pense que l'Angleterre est pour les Anglais. Si les étrangers viennent ici ils doivent se conformer au système ».

De leur côté, les médias ont longtemps dépeint le carnaval de Notting Hill comme un événement violent et dangereux, en particulier après les nouvelles violences de 1987 durant lesquelles la plupart des quotidiens ont tour à tour fait campagne pour l'interdiction du carnaval, ou pour le moins sa privatisation: " Il n'y a aucune raison pour que le carnaval dépende des dons d'institutions telles que le Arts Council, la Commission for Racial Equality et les autorités locales, pouvait-on lire dans l'édition du $1^{\text {er }}$ août 1988 de The Independant, journal pourtant réputé modéré. Le sponsorship, la vente des droits télé et la mise en œuvre d'événements complémentaires pourraient être une mine d'or et générer des fonds dans une zone de déchéances urbaines ». La mise en place du NHCT et le recul des violences à partir du début des années 1990, ont cependant contribué à déplacer le traitement du carnaval des pages «Faits Divers » aux rubriques «Loisirs» ou «Culture», apportant une connotation beaucoup plus respectable à l'événement. 


\section{Le carnaval comme nouvelle forme de vivre ensemble}

Depuis les résidents tourmentés par le bruit et le désordre au débat sur la sécurité, depuis l'abandon des subventions à l'adoption de méthodes managériales, de la célébration communautaire à celle d'une événement multiculturel, le carnaval est devenu en 20 ans (1970-1990) un problème complexe de politique publique. Aujourd'hui, et comme le montre l'énumération précédente, il peut inclure les facteurs les plus localisés parking automobile, financement des costumes, identité individuelle - et les considérations les plus générales - citoyenneté, néo-libéralisme, nationalisme: comment un tel événement peut-il dépasser les intérêts individuels et donner de la substance à une politique du bien commun ? Afin de clarifier cette question, nous devons commencer par identifier comment les différentes orientations du carnaval ont été politisées au point de s'identifier avec le programme de la New Left au tournant des années 1980. Nous examinerons ensuite les possibilités de modelage de ces revendications contre-culturelles dans des ordres de légitimation variés, depuis les plus générales comme celles portées par la GLA aux plus particulières comme celles défendues par certaines associations de défense du carnaval.

\subsection{Les critiques sociale et culturelle portées par le carnaval}

Considérons tout d'abord la capacité des arguments du carnaval à apporter des changements radicaux. A partir des années 1970, l'insatisfaction à l'égard du Parti travailliste et le développement d'une critique radicale attentive au développement des pratiques sous-culturelles favorise un regain d'intérêt à l'égard du carnaval. La culture populaire est désormais largement perçue comme une arène centrale où la lutte politique peut prendre place, et le carnaval est en ce sens progressivement conceptualisé par les théoriciens du CCCS de Birmingham comme une métaphore des transformations sociales et culturelles de la société britannique (Harris 1992). De fait, plongeant ses racines d'une part dans l'histoire de l'esclavage et d'autre part dans les luttes anti-racistes des noirs immigrés à Londres, le carnaval de Notting Hill est aisément présenté comme une critique de la société britannique, et plus largement de l'impérialisme capitaliste. Il apparaît de ce point de vue comme une occasion « révolutionnaire » dans laquelle la résistance au «pouvoir blanc», et plus 
largement au système capitaliste peut s'exprimer dans toute son intensité (Hebdige 1979; Hall 1996). Théorisée par les intellectuels de la «nouvelle gauche » britannique, la rébellion des jeunes noirs qui s'exprime lors du carnaval de 1976 semble dès lors démontrer une continuité fondamentale entre expression culturelle et action politique. Parmi les nombreuses contributions des théoriciens culturels de la New Left, nous retiendrons les analyses de Paul Gilroy, l'un des plus célèbres collaborateur du CCCS, et plus particulièrement un article publié pour la première fois en 1990 où il tente d'analyser les dimensions culturelles et sociales des mouvements urbains noirs. Pour P. Gilroy (1993), « les formes artistiques ont produit et nourrit une communauté interprétative hors de l'orbite de la politique formelle dans une longue séquence de luttes qui ont été irréductiblement et simultanément à la fois culturelles et politiques»(p. 32). De fait, c'est précisément «dans la rencontre entre les habitants noirs et leurs voisins bancs des quartiers populaires, que la culture noire est devenue une culture de classe » (p. 34). C'est pourquoi la politique de ces mouvements, et du carnaval de Notting Hill en particulier, peut se manifester à la confluence de trois critiques du capitalisme «qui forment une politique globale mais nonprogrammatique qui a nourri les populations noires résidant en GrandeBretagne et leurs voisins blancs des quartiers populaires » (p. 35).

La première critique met en question les promesses de la rhétorique démocratique de la légalité capitaliste: «Les institutions légales sur lesquelles repose l'ordre de l'autorité publique de Babylone ne fournissent pas des droits égaux à tous. La version de la justice qu'ils colportent est partiale et inséparable du système des intérêts économiques que la légalité capitaliste garantit simplement» (Ibid, p. 36). De ce point de vue, si le carnaval a souvent été le lieu d'une confrontation violente avec la police, il est avant tout marqué par la volonté de parodier la société d'accueil et de contribuer au renversement des valeurs et des positions, comme l'exprime ici un costumier cité par The Independant (3 août 1988) : «Le carnaval est une affaire de masques - une affaire de déguisement. Nous sommes tout le temps en train de nous déguiser; nous présentons un masque au monde lorsque nous nous habillons chaque jour. Regardez la police, regardez les soldats, regardez la famille royale - ils jouent tous un rôle, se déguisant pour jouer ce qu'ils veulent être ». La dérision est en outre au cœur de la tradition du Calypso, qui s'est développé à Trinité comme un élément de critique politique (Cowley 1996). La politique du carnaval apparait ici comme une 
opportunité de développer une contre-culture dans les interstices du contrôle social et politique.

La seconde critique s'attaque à l'expérience du travail, à ses conditions et à sa division sous le capitalisme : «Elle conduit à une critique du productivisme - l'idéologie qui voit le développement des forces productives comme une condition indispensable pour atteindre la liberté » (Gilroy 1993, p. 36). En ce sens, comme le soulignait un fabriquant de costumes, «A la différence du travail capitaliste, ici tu décides de ce que tu crées; il n'y a pas d'horaires ou de contremaîtres. Tu rends ta création spéciale, chargée de tes propres trésors et parlant ton langage » (City Limits, 18-25 août 1988). La résistance de la majorité des joueurs de steel bands antillais à toute rationalisation ou standardisation de la production des pans est de ce fait particulièrement frappante, que ce soit dans leur fabrication et leur entretien ou dans l'organisation et la coordination musicale du groupe (Cohen 1993). La modernisation est ici accusée d'éloigner le joueur de son instrument, de standardiser le pan et de contribuer à restreindre l'expérimentation créative rendue possible par l'individuation de l'instrument. En devenant rationnellement organisée, la musique cesserait d'être «populaire » et son impact politique serait perdu.

La troisième critique se concentre enfin sur l'importance de l'histoire, dépeinte «comme un antidote à la suppression de la perception temporelle sous le capitalisme » (Gilroy 1993, p. 37). La mise à jour/mise en scène de l'histoire du groupe doit permettre de révéler les sources d'injustice, à conscientiser et renforcer la communauté pour finalement résister à l'oppression dont les noirs font l'objet. A ce niveau, le carnaval peut-être perçu comme un instrument au service du développement communautaire, avec ses réseaux d'amitié interpersonnels. Le carnaval aide à la mobilisation de la communauté et la communauté développe le carnaval. La forme élémentaire du pan et du steel band nécessite par exemple une interaction de face à face intensive avec les joueurs, comme l'explique Pepe Francis, leader de l'Ebony Steel Band: "Les steel bands et le carnaval peuvent faire ce que les politiciens ne parviennent pas, relier les gens entre eux dans une bonne humeur permanente. La liberté est si électrifiante que les préjugés n'ont pas leur place. Le carnaval est la meilleure forme de relations raciales qui soit» (entretien, 25 avril 2000). Le carnaval fournit ici un contexte unifiant pour le partage et la célébration d'expériences collectives, 
où l'interaction entre les interprètes et les spectateurs dissout la disjonction (dénoncée comme euro-centrique) entre l'art et la vie, le dedans et le dehors, le privé et le public.

\subsection{Le carnaval au service du développement local}

Transfiguré par l'analyse des théoriciens de la New Left, le carnaval est-il en mesure d'influencer voire de transformer le système en place en imposant un nouveau principe de justification et d'évaluation des cultures minoritaires dans la société britannique ? Dans un contexte de responsabilisation croissante des gouvernements locaux (managérialisation des finances locales imposée par les lois Thatcher, processus de dévolution engagé par Tony Blair) et de raréfaction de finances locales, ce qui participait d'abord d'une bataille politique se transforme progressivement en une volonté de rentabiliser au mieux les potentiels locaux (Imrie and Raco 2003). La critique du système diffusée par les militants de la New Left à travers leur instrumentalisation de la figure du carnaval peut-elle alors être articulée à des logiques d'émancipation sociale et culturelle sans être absorbée par les logiques de développement de la «cité globale»? Afin de tester la validité de l'hypothèse d'une « cité carnavalesque », il nous faut à la fois questionner les rapports entretenus par les valeurs portées par le carnaval et les modes d'évaluation des politiques urbaines actuellement en vigueur, et vérifier que la ligne d'argument du carnaval déploie une spécification du lien politique en mesure de créer un accord légitime.

Confrontée à une compétition territoriale croissante, les villes doivent aujourd'hui présenter un profil efficace en matière de gestion économique, une efficacité de plus en plus associée à leur capacité à maintenir une certaine cohésion sociale et culturelle (Le Galès 2003). De tels lieux doivent présenter des images positives d'elles-mêmes au monde extérieur. C'est à ce type d'ambition qu'a répondu la création en 2000, par le Premier ministre travailliste Tony Blair, de la Great London Authority (GLA), avec la volonté d'encourager une coordination globale des problématiques urbaines dans une ville jusque là marquée par de très fortes dissensions en matière de développement urbain. L'accession à la tête de cette nouvelle assemblée de Ken «Le Rouge », ancien porte-voix de la New Left lorsqu'il était maire du GLC de 1981 à 1985, résonne pour sa part 
comme le triomphe d'une conception «multiculturelle» de l'avenir de Londres (sur la politique du GLC, cf. Bianchini 1987 ; sur les enjeux du GLA, Pilmott and Rao 2002). Mais tout en reconnaissant les valeurs du carnaval comme un événement significatif pour tous les Londoniens, le nouveau maire de Londres ne s'en est pas moins engagé dans son programme électoral à « encourager une organisation efficace et sécurisée du carnaval de Notting Hill » (Ken For London 2000). Dès la fin des années 1980, la police avait elle-même engagé un processus de consultation des organisateurs pour prévenir les débordements. Face aux dénonciations récurrentes et fortement médiatisées, y compris au niveau international, des brutalités attribuées à la police londonienne contre les carnavaliers, les autorités policières ont mis en place des groupes de consultation locale pour discuter de problèmes de "sécurité publique», une façon d'éviter de focaliser l'attention sur le conflit police/noirs. Les médias ne s'y sont pas trompés, puisqu'ils présentent désormais le carnaval comme un «mélange de cultures» (The Voice, 21 août 2000, supplément carnaval), «un événement joyeux et multiculturel (en dépit d'assertions persistantes qu'il s'agit d'un événement antillais)... » (The Guardian, 2 septembre 1989).

Dans une étude de l'impact économique du carnaval, la London Development Agency (LDA), créée en 2000 par le gouvernement Blair pour prendre la tête du développement économique et de l'activité de régénération urbaine de la capitale, rapporte que $71,5 \%$ des visiteurs ( « toutes catégories d'ethnicité ») déclarent que la culture caribéenne manifestée par le carnaval fait partie de l'identité britannique, tandis que 94\% se disent «d'accord» voire « tout à fait d'accord » avec l'idée que le carnaval est un événement clé de la vie culturelle londonienne (LDA, 2003, p. 17). Pour la LDA, il ne fait donc aucun doute que cette bonne image se doit être développée dans la publicité et le marketing : «Le Carnaval est un événement culturel commercialisable. Dans les années récentes, le Carnaval a été promu comme un événement culturel clé dans le calendrier de Londres, créant une «marque de fabrique » [brand] grâce à laquelle un nombre croissant de consommateurs seront attirés » (ibid. p. 36). Le rapport de la LDA évalue les effets économiques du carnaval sur l'économie londonienne à plus de 93 millions de livres chaque année, tandis que sa préparation et son déroulement constitueraient un gisement potentiel de 3000 emplois à temps plein. De ce point de vue, si le carnaval est en mesure de mettre en scène l'intégration des noirs en Grande-Bretagne, le développement d'une 
économie dite «ethnique », où chacun peut en profiter pour vendre des produits (à condition néanmoins de payer une licence), contribue en outre au renforcement (empowerment) de la communauté locale. Comme l'audit de Coopers \& Lybrand le soulignait dès 1988, «Une grande partie de la communauté noire de Notting Hill (et à travers Londres) valorise le carnaval comme l'événement de l'année mais, de façon plus importante, comme l'événement noir organisé par des noirs dans un pays où la "propriété » et l'influence des noirs sont très limitées » (Coopers \& Lybrand, 1988, p. 2).

De ce point de vue, l'intérêt du rapport commandé par la LDA est certainement de mettre l'accent sur «les effets économiques multiplicatifs de la contribution créative» du carnaval, susceptibles de générer bien davantage de bénéfices sociaux et économiques. A ce niveau, le carnaval de Notting Hill fournit un contexte privilégié pour le développement actif d'une grande variété de compétences «créatives » pour des groupes et des individus autrement absents de ces secteurs ${ }^{3}$. Pour les auteurs du rapport, il s'agit de se concentrer sur «la transférabilité des compétences créatives développées dans le cadre du Carnaval, comme dans la mode ou le théâtre ; mais aussi sur le potentiel d'une variété de secteur liées aux industries créatives pour ajouter de la valeur (et tirer les enseignements) du processus du carnaval» (LDA, 2003, p. 37). Ces conclusions sont reprises dans le rapport final du Carnival Review Group commandé par le GLA et publié en juin 2004. La culture acquiert dès lors un rôle stratégique, qui va bien audelà d'une lutte symbolique pour le pouvoir. Dans le cadre de la mise en place d'un "Cultural Strategy Group » chargé d'établir un agenda culturel pour Londres, un Carnival Officer a été nommé pour réfléchir aux potentialités offertes par le carnaval. Désormais, le carnaval de Notting Hill doit être pensé comme un «événement de classe internationale » (entretien, avec Michael Campbell, Senior Cultural Projects Officer, GLA, 29 août 2002) susceptible de refléter non seulement la place et le rôle des AfroAntillais, mais de valoriser également l'image d'une ville multiculturelle et créative. En désignant tout autant le patrimoine et les œuvres susceptibles d'être valorisées à des fins touristiques que les normes et les valeurs

\footnotetext{
${ }^{3}$ Une liste toujours plus longue de secteurs industriels - la musique, le cinéma, le théâtre, la publicité, le design, l'architecture, etc. - ont été associés à la «créativité » humaine par le New Labour, tandis que pratiquement tous les autres y sont reliés indirectement par le design, la publicité et le marketing des produits (cf. DCMS, 1998).
} 
partagées par les habitants, la culture (communautaire et artistique) devient ici un élément essentiel de la stratégie économique des élites urbaines.

\subsection{La « carnavalisation » et ses limites : le renouvellement de la} critique

Nous avons vu comment les arguments contre-culturels portés par la figure du carnaval pouvaient être fondus dans un ordre de légitimation plus général. Mais quelles sont les conséquences de l'introduction de ce nouveau principe de justification sur la grammaire commune ? L'orientation vers la justification suppose que les personnes impliquées dans le jugement soient capables d'extraire leurs activités de leurs intérêts particuliers afin de montrer l'étendue de l'intérêt général auquel ils participent. Un des principes de la grammaire commune qui s'applique aux différentes formes de justification réside par conséquent dans une communauté de référence composée de personnes d'égale dignité susceptibles de faire entendre leur voix et de mettre en question les ordres de grandeurs dominants (Boltanski et Thévenot 1991). Or certains arguments et certains types de preuves et d'expertises identifiés dans le cadre du carnaval de Notting Hill suggèrent un mouvement inverse, du général vers le particulier, vers un renouveau de la critique, plus sensible aux particularismes collectifs et/ou individuels.

La réhabilitation progressive du quartier de Notting Hill, principalement en raison de la proximité de l'hyper-centre et d'un certain nombre de quartiers résidentiels dans un contexte de pression foncière croissante, a rapidement repoussé les classes populaires (en grande partie des noirs) vers les quartiers périphériques de Lambeth, Hackney, Brent ou même Lewisham. Or, ce processus de remplacement des classes populaires par des classes moyennes, d'augmentation du prix du foncier, de transformation du bâti et, finalement, l'arrivée de nouvelles modalités de vivre le quartier n'a pas été sans conséquence sur le Carnaval. En effet, les intérêts matériels des résidents ont encouragé une régulation de plus en plus intense de son organisation et la police n'a pas tardé à être accusée de défendre les intérêts des nouveaux habitants. Pour l'Association for a People's Carnival, le carnaval de Notting Hill est ainsi devenu le «Carnaval de la Police»: «Les groupes de carnaval ne sont plus en mesure de mettre en scène librement leur art et de s'amuser dans les rues. Non seulement la police leur dicte la route à 
suivre mais également qui est autorisé à danser avec le groupe!» (Association for a People's Carnival, p. 1). Tout au long des années 1980, la sécurisation du carnaval est dénoncée par les leaders du CAC et, avec elle, les transformations subies par l'événement, à l'image des critiques formulées par Alex Pascall en 1988 : «Il y a eu beaucoup de tension dans les rues parce que la police a changé la nature du carnaval. Nous ne pouvons plus avoir le carnaval auquel nous étions habitués » (cité par The Independant, 30 août 1988). Aujourd'hui, la nouvelle politique du GLA est pareillement dénoncée comme contribuant à détacher de plus en plus l'organisation et les objectifs du carnaval de la communauté noire pour les rapprocher des goûts et des besoins des touristes et des amateurs de festivals.

L'amertume des leaders historiques du carnaval de Notting Hill s'apparente de fait à un sentiment de dépossession. Pour eux, les exigences des pouvoirs publics - et avec eux des «bourgeois noirs » qui collaborent avec l'Etat - ont contribué à stériliser et calibrer le carnaval pour mieux le vendre sur le marché des biens touristiques et culturels. Le nouveau comité d'organisation présidé par Claire Holder est particulièrement critiqué : «Le nouveau groupe, connu aujourd'hui sous le nom de Notting Hill Carnival Trust a cherché à maximiser ses revenus à travers le sponsorship. Mais, comme je le découvrais alors dans les pages économiques du journal, il était très difficile de vendre quelque chose de «noir» au public blanc, et l'événement a été repositionné avec des slogans tels que «harmonie interculturelle », «tolérance raciale », « cosmopolitisme »... le sort en était jeté. En un sens, ça marche : lentement, les sponsors qui ne voulaient pas mettre un centime il y a quelques années dans quoi que ce soit de «noir» commencèrent à ouvrir leurs portefeuilles. (...) Reste que les bailleurs de fonds ne ciblent pas nécessairement les noirs - leur audience est plutôt les blancs dans le vent qui sont désormais majoritaires dans la foule du carnaval » (Joseph Harker, «The Carnival is over », The Guardian, 26 août 2000).

Comme le déplorait également un éditorialiste de l'hebdomadaire noir New Nation: le carnaval de Notting Hill «n'est plus «noir», il est devenu «urbain»» («When did «black» become such a dirty word», 21 août 2000). A la différence de la critique classique qui dénonçait les risques de standardisation, c'est ici la codification de la culture noire qui est dénoncée comme un facteur de marchandisation de la différence elle-même, 
ce qui n'était évidemment pas possible dans la cadre d'une production standardisée (Cashmore 1997). De ce point de vue, l'authenticité proclamée des steel bands et des sound systems, qui reposait jusque là sur l'impossibilité de reproduire leurs performances, devient tout aussi marchandisable dans la mesure où la codification permet de conserver quelque chose de la similarité qui donnait de la valeur à l'original (Boltanski et Chiapello 1999, p. 537-541).

Gloria Commins, secrétaire de la London Costume and Mask Association (LCMA) et membre de l'assemblée générale du NHCT, le soulignait dès 1988 : «Dès que des critiques sérieuses émergent, la question des blancs contre les noirs est mise sur le tapis » (The Independant, $1^{\text {er }}$ Août 1988). De fait, à la suite du rapport réalisé par les consultants Coopers \& Lybrand, le Carnival Anti-Apartheid Coordinating Committe accusa ces derniers de travailler avec le régime sud-africain : "Une telle collaboration affaiblit la crédibilité d'un tel rapport », s'empressèrent de déclarer certains militants du carnaval (cité par The Voice, 16 août 1988). Une présomption de racisme qui agit ici comme un argument pour conserver la gestion du carnaval éloignée des principes généraux : «Je ne pense pas que les maîtres puissent venir et dire aux esclaves comment exprimer leurs sentiments durant le carnaval, déclarait Alex Pascall (...). Aucun carnaval dans le monde n'a jamais été initié sur des bases économiques et le carnaval de Notting Hill n'est pas différent (...). Il n'y a aucune raison pour que nous nous préparions à payer une autorisation. C'est notre carnaval. C'est notre culture et cela l'a toujours été. Nous vivons ici » (cité par City Limits, 18-25 août 1988).

Ce type d'argument, proche de l'invocation du «droit à la différence », vise ici à prévenir toute forme de «lactification» (selon le mot de Frantz Fanon) présumée du carnaval, comprise comme une tentative larvée de transformation de «l'esprit» du carnaval (que seule serait en mesure de garantir la communauté afro-antillaise) et qu'une modernisation irréfléchie menacerait, si l'on en croit le fondateur du magazine Black Britons : «L'esprit de Notting Hill a été perdu lorsqu'il a commencé à s'occuper de l'harmonie interculturelle. (...) En réalité, de nos jours, seulement une petite proportion de «fêtards» sont noirs, et dans un événement qui était organisé par et pour les Afro-Antillais, seulement un petit noyau crée désormais la musique et l'atmosphère, et tout cela pour le 
bénéfice d'une audience séparée... pareil à un concert, mais avec un peu plus d'artistes noirs et où les clients viennent gratuitement. Tout comme Notting Hill lui-même, le carnaval a été "gentrifié », un endroit où se rendent les jeunes branchés pour montrer qu'ils sont «cools» »(Joseph Harker, op. cit).

\section{Conclusion}

Les diverses formes de rapprochement entre les revendications portées par le carnaval et les politiques publiques londoniennes ont progressivement remis en cause la césure (longtemps maintenue par les conservateurs) entre l'ordre social et politique et un ordre "carnivalesque » où s'exprimerait librement les cultures minoritaires. Les justifications qui président aujourd'hui à la promotion du carnaval reposent en effet en grande partie sur la thématique de l'hybridation et du métissage, non seulement avec l'idée d'épingler les fautes et les insuffisances des modes d'évaluation traditionnels du lien national, mais également pour contester leur légitimité à assurer le bien commun et à discréditer sa capacité d'évaluation. En ce sens, le pouvoir du carnaval ne repose pas seulement sur une simple renversement temporaire du haut par le bas, mais dans l'émergence de formes hybrides, «grotesques » : c'est le pouvoir de classifier qui est ici mis en question (Hall 1996). La critique du capitalisme développée dans le cadre du carnaval ne se contente donc pas de revendiquer l'égalité, elle met en question la notion générale d'essentialisme, de race et d'ethnicité par la rencontre physique et le mélange des individus. Un métissage célébré par le carnaval qui annonce l'établissement d'un nouvel ordre de grandeur entre les personnes et les choses qui peuplent la cité globale. Dans cette nouvelle cité, la personne est grande parce qu'elle est métissée, autrement dit parce qu'elle accepte de rentrer en contact avec les autres et de partager leur identité. Le carnaval permet de penser l'identité en termes de contingence, d'indétermination et de conflit; une identité en mouvement plutôt qu'un absolu de nature ou de culture qui se prête merveilleusement bien aux nouvelles exigences de la compétition interurbaine et de la société en réseau décrites notamment par Manuel Castells (1999).

Mais la généralisation des intérêts au profit d'une cité qui tient son enrichissement de sa capacité à capter et brasser toutes ses ressources 
potentielles n'en est pas moins vécue par certains comme une menace vis-àvis de leur intégrité identitaire. La gentrification progressive du quartier de Notting Hill, la marchandisation de la «culture noire », ou encore la présence de plus en plus massive d'un public et d'artistes blancs conduit certains acteurs à revendiquer une certaine «authenticité » du carnaval, et finalement à engager un mouvement inverse du général vers le particulier. La défense du carnaval est dès lors orientée vers un renouveau de la critique : la césure est renforcée et traduite en une différence de sensibilité entre les noirs et les blancs à l'image de certains DJ's qui incitent leur auditoire à «stay black» ou « keep it real ». Plus précisément, il est frappant de noter qu'après la création du NHCT en 1989, les militants du CAC recherchèrent une légitimité en proclamant leur désir de «revenir au peuple » : «Le carnaval appartient aux gens ordinaires, ils l'ont inspiré et l'ont guidé dans son développement, et ma loyauté va à eux (...). Le carnaval a été initialement créé par la communauté caribéenne dans les rues de Notting Hill et a été ensuite apprécié par toute la population » (Alex Pascall, Caribbean Times, 25-31 août 1989). Comme l'indique également l'intitulé même de l'association «for a People's Carnival», une des caractéristiques du critère de justification des défenseurs du carnaval est d'en appeler au peuple pour mieux s'opposer à l'élite et à la haute culture. La culture populaire est ici perçue comme le site de traditions alternatives tandis que l'authenticité noire se doit d'être recherchée du côté des classes laborieuses qui ont seules résisté à l'assimilation et à la naturalisation de l'infériorité des noirs (Favor 1999). On comprend alors que, pour survivre, il est nécessaire de rester marginal : effacer la frontière, comme y invite la cité carnavalesque, c'est risquer de s'effacer soi-même (Barth 1969). Or en contribuant à mettre au second plan les inégalités socio-économiques au profit d'un multiculturalisme rassembleur et consensuel, la promotion du carnaval par les autorités londoniennes court le risque de favoriser au contraire le réveil d'un besoin d'affiliation identitaire chez des populations qui n'auraient ni l'envie ni les moyens de se «connecter» aux exigences de la cité globale. 


\section{BIBLIOGRAPHIE}

ARNAUD, Lionel, 2005. "Le carnaval comme événement politique ? Les expressions culturelles minoritaires à l'épreuve de la politisation dans le carnaval de Notting Hill et le défilé de la Biennale de la danse de Lyon », p. 177-208, in: L. Arnaud et C. Guionnet (dir.). Les frontières du politique : enquêtes sur les processus de politisation et de dépolitisation. Rennes: Presses Universitaires de Rennes.

ASSOCIATION FOR A PEOPLE'S CARNIVAL, undated. 'Police Carnival' 1989 : a report on the 1989 Notting Hill Carnival.

BAKHTIN, Mickael, 1970. L'oeuvre de François Rabelais et la culture populaire au Moyen Âge. Paris : Gallimard.

BARTH, Frederick (ed.), 1969. Ethnic groups and boundaries : the social organization of Culture Difference. London : Georg Allen and Unwin.

BIANCHINI, Franco, 1987. "GLC - RIP : Cultural policies in London 19811986”, New Formations, n¹.

BIANCHINI, Franco, 1989. "Cultural problems and urban social movements : the response of the "New Left" in Rome (1976-1985) and in London (1981-1986)", in : P. BRAMAHAM, I. HENRY, H. MOMMAAS and H. VAN DER POEL (eds). Leisure and urban processes : critical studies of leisure policy in Western European cities. Londres : Routledge \& Kegan Paul.

BOLTANSKI, Luc et CHIAPELLO, Eve, 1999. Le nouvel esprit du capitalisme. Paris : Gallimard.

BOLTANSKI, Luc et THÉVENOT, Laurent, 1991. De la justification. Paris : Gallimard.

CARNIVAL AND ARTS COMMITTEE, June 1988. Organization review, Coopers \& Lybrand.

CASHMORE, Ellis, 1997. The black culture industry. London : Routledge.

CASTELLS, Manuel, 1999. L'ère de l'information. Paris : Fayard. 
CHASE, Louis, 1978. Notting Hill Carnival, street festival. Londres : Interlink Longraph Ltd.

COHEN, Abner, 1991. 'Drama and politics in the development of a London Carnival', p. 170-202, in : WERBNER, Pnina and ANWAR, Muhammad (eds). Black and ethnic leaderships : the cultural dimensions of political action. London : Routledge.

COHEN, Abner, 1993. Masquerade politics : exploration in the structure of urban cultural movements. Oxford : Berg.

COOPERS \& LYBRAND, 1988. Carnival and Arts Committee Organisation Review, dactylographié.

COWLEY, John, 1996. Carnival, canboulay and calypso : traditions in the making. Cambridge : Cambridge UP.

DEPARTMENT OF CULTURE, MEDIA AND SPORTS (DCMS), 1998. Creative industries mapping document. Londres : HMSO.

FAVOR, J. Martin, 1999. Authentic blackness. Durham and London : Duke University Press.

GILROY, Paul, 1993. 'One nation under a groove', p. 19-48, in : Small Acts. London : Serpent's Tail.

GUTZMORE, Cecil, 1993. 'Carnival, the State and the Black Masses in the United Kingdom', p. 207-232, in : Winston James and Clive Harris (eds.). Inside Babylon : the caribbean diaspora in Britain. London : Verso.

HALL, Stuart, 1996. 'For Allon White. Metaphors of transformation', p. 287305, in : David Morley and Kuan-Hsing Chen (eds). Stuart Hall : critiqueal dialogues in cultural studies. London : Routledge.

HARRIS, David, 1992. From class struggle to the politics of pleasure: the effects of gramscianism on Cultural Studies. London : Routledge.

HASSENTEUFEL, Patrick, 1995. 'Les groupes d'intérêt dans l'action publique : l'Etat en interaction', Pouvoirs, n ${ }^{\circ} 74$, p. 155-168.

HEBDIGE, Dick, 1979. Subculture, the meaning of style. Londres : Routledge. 
HOWLETT, Mickael, 1994. 'Policy paradigms and policy change : lessons from the old and new canadian policies towards aboriginal peoples', Policy Studies Journal, vol. 22, n . 4, p. 631-651.

IMRIE, Rob and RASCO, Mike, 2003. Urban renaissance? New labour, community and urban policy. Bristol : The Policy Press.

JOBERT, Bruno et MULLER, Pierre, 1987. L'Etat en action. Paris : P.U.F.

KEN FOR LONDON, 2000. Ken Livingstone's manifesto for London, dactylographié.

KENNY, Michael, 1995. The First New Left : british intellectuals after Stalin. Londres : Lawrence and Wishart.

LAFAYE, Claudette and THÉVENOT, Laurent, 1993. 'Une justification écologique ? Conflits dans l'aménagement de la nature', Revue française de sociologie, vol. 34, $\mathrm{n}^{\circ}$ 4, p. 495-524.

LE GALES, Patrick, 2003. Le retour des villes européennes. Paris : Presses de Sc Po.

LONDON DEVELOPMENT AGENCY, 2003. The economic impact of the Notting Hill carnival. Londres : Mayor of London.

LONDON RESEARCH CENTRE, 1994. London's ethnic minorities : an analysis of 1991 Census results. Londres : LRC.

MCCOOL, Daniel, 1989. 'Subgovernments and the Impact of Policy Fragmentation and Accommodation', Policy Studies Review, vol. 8, ${ }^{\circ} 2$, p. 264-287.

NOTTING HILL CARNIVAL, 1999. Safety Advisory Group, Minutes of meeting held on 1st July 1999.

OFFERLE, Michel, 1998. Sociologie des groupes d'intérêt. Paris : Montchrestien.

PILKINGTON, Edward. Beyond the mother country: West Indians and the Notting Hill white riots. London : I.B. Tauris, 1988. 182 p.

PIMLOTT, Ben and NIRMALA, Rao, 2002. Governing London. Oxford : Oxford University Press. 
PRYCE, Everton A., 1995. 'The Notting Hill Gate Carnival. Black politics, resistance, and leadership 1976-1978', Caribbean Quarterly, vol. 21, n 2, p. $35-52$.

ROACH, Larry, 1992. 'The Notting Hill Carnival : an exercise in conflict resolution', p. 101-106, in : Tony F. MARSHALL (ed.). Community, Disorders and Policing. London: Whiting and Birch.

SASSEN S., 1991. The global city. New York, London, Tokyo, Princeton : Princeton University Press. 398 p.

THE ROYAL BOROUGH OF KENSINGTON AND CHELSEA, CITY OF WESTMINSTER, METROPOLITAN POLICE, 2000. Notting Hill Residents' information bulletin. 


\title{
RESUME \\ Une cité carnavalesque? Le carnaval de Notting Hill à l'épreuve des politiques de développement urbain
}

En se fondant principalement sur les discours et les débats qui ont accompagné le développement du carnaval depuis la fin des années 70, cet article propose une analyse des processus sociaux qui président aujourd'hui à la promotion sociale et culturelle du carnaval de Notting Hill par le gouvernement local. Il présente d'une part, les affrontements d'acteurs auxquels le carnaval a donné lieu et examine, d'autre part, les médiations par lesquelles les références à la «cité globale » (qui désigne ici non seulement l'entité urbaine formée par la ville de Londres, mais plus largement l'univers qui lui sert aujourd'hui de justification) permettent le passage du particulier au général et finalement la légitimation du carnaval comme outil au service du développement social de la ville de Londres.

\begin{abstract}
A carnivalesque city ? Notting Hill Carnival put to the test by politics of urban development

Principally founded on the discourses and debates going with the development of the Carnival since the end of the seventies, this article analyses of the social processes which prevails through the social and cultural promotion of the Notting Hill Carnival by the local government today. On one hand it sets out the confrontations of actors that the Carnival gave rise to, on the other hand it examines the arbitration by which the references to a "global city" (designating not only the urban entity of the city of London but the whole universe serving its justification), allow the passage from the particular to the general and finally the legitimation of Carnival as a tool for the social development of the city of London.
\end{abstract}


